\title{
Design and Optimization of Directive Circular Patch Antenna with Asymmetric Pixels Using Genetic Algorithm
}

\author{
Sinan Doğusan
}

\begin{abstract}
In this study, a low-profile, high directive circular microstrip antenna was designed for $5.8 \mathrm{GHz}$ ISM band applications. In the design, the GA / MoM approach based on the Method of Moments (MoM) integrated with the Genetic Algorithm (GA) optimization method was used. The simulations of the best first and second antennas obtained as a result of the optimization process were made using the ANSYS HFSS software program. According to the simulation results, it was determined that the input reflection coefficient of both antennas was below -10 $\mathrm{dB}$ in the ISM band where the antenna was operating and had maximum directivity. It was observed that the radiation characteristics of both antennas obtained with the GA / MoM approach were steady-state in the operating band. It was concluded that asymmetric pixelation used in this method can be used in the design of antennas with different geometries.
\end{abstract}

Index Terms - Genetic Algorithm, Method of Moments, Microstrip Patch Antenna, Optimization

\section{INTRODUCTION}

$\mathrm{R}$ ECENTLY, WITH the increase in performance levels demanded in wireless communication technology, the need for low-profile and high-performance antenna design throughout the operating band is increasing. This need has led to the emergence of new developed solution methods different from traditional design methods [1,2]. Because traditional design methods used in antenna design problems are insufficient in solving complex problems.

In line with this rapid development, numerical solution methods are used in solving complex electromagnetic problems to obtain more accurate results in a shorter time [3-5]. In addition to these methods, optimization algorithms also play an important role in solving antenna design problems. As the complexity of the antenna design problems to be solved increased, optimization algorithms (Stochastic, Deterministic, Heuristic, Mathematical) that can discover many powerful, innovative and large solution sets that can be used by integrating numerical methods have been developed to overcome these problems in a short time [6-8]. One of the most

SÍNAN DOĞUSAN is with Department of Electrical-Electronics Engineering Inonu University, Malatya, Turkey,(e-mail: sinandogusan@gmail.com).

(iD https://orcid.org/0000-0002-2207-8904

Manuscript received February 20, 2021; accepted June 13, 2021. DOI: $\underline{10.17694 / \mathrm{bajece} .883468}$ preferred of these optimization algorithms is the Genetic Algorithm (GA) that models biological processes inspired by Darwin's theory of evolution. GA is an heuristic stochastic global optimization algorithm based on the principle of survival of the best [9]. Since GA has the property of converging to the best result, it is highly preferred in antenna design problems [10-17].

GA searches for the most suitable substructure with the radiation characteristic targeted for the design by optimizing any physical properties of an antenna to be designed. One of the most important of these is the problem of finding the best current distribution on the surface for a specified purpose. By using GA in these problems, removing some pixels from the radiating pixelized surface of the antenna during the optimization process, the best surface current distribution with the targeted radiation characteristics is obtained. In previous studies, symmetric pixel selection for such problems has been perfectly adapted to quadratic surfaces and it was observed that the radiation parameters of the antennas had as a result of optimization were quite good $[18,19]$. However, if surfaces with circular or non-quadratic geometry are wanted to be separated into symmetrical pixels for optimization, gaps will occur at the end regions of the surface. As a result, analysis will have to be applied in a more limited area. Due to its inclusion in a limited surface area optimization process, obtaining the antenna with the targeted radiation parameters will be difficult. To overcome this problem, the empty surface area can be filled by creating smaller symmetrical pixels. Hereby, an antenna with aimed radiation parameters can be achieved by increasing the surface area to be included in the optimization thanks to the increasing number of pixels. However, an increase in the number of pixels will also cause an increase in the size of the moment matrix. Therefore, the increase in the time spent for optimization and in memory used should be considered.

For this reason, in order to get the antenna with the targeted radiation parameters, a new pixel architecture has emerged that will create a lower-dimensional moment matrix by dividing the surface to be optimized into a minimum number of pixels and enable analysis on all geometric surfaces. So as to meet this need, the method of asymmetric pixelation was suggested for the design of antennas that have circular or non-quadratic geometries in this study. The method of shaping the radiating 
surface of the antenna to be included in the optimization into asymmetric pixels enables full surface analysis without any restrictions on the surface areas. Thanks to this method proposed, it is possible to design the antenna with the radiation parameters targeted by the designer in a shorter time using the minimum number of pixels.

In this article, a circular patch antenna with high directivity and low input reflection coefficient characteristics was designed. For this purpose, a $5.8 \mathrm{GHz}$ ISM (Industrial, Scientific, and Medical) frequency band $50 \Omega$ coaxial probe and an antenna model fed from the center [20] were discussed. To obtain the antenna with these characteristics, the GA / MoM optimization method based on the use of Genetic Algorithm (GA) integrated with Method of Moments (MoM) was used [1]. In order to apply this technique, the lengths of the main circular radiating surface were first determined. Then this radiating conductive surface was then divided into asymmetric pixels to be optimized and optimization was made for each pixel. The results achieved were shown in graphics and a table.

\section{MATERIAL AND METHODS}

\section{A. Antenna Geometry}

The proposed antenna geometry is shown in Figure 1. The antenna that designed to work in the $5.8 \mathrm{GHz}$ ISM band consists of 406 asymmetrical copper parts with a radiating surface of $0.02 \mathrm{~mm}$ in height. The radius of the antenna was determined $5.1 \mathrm{~cm}$, which is equivalent to a wavelength, and the dielectric material height was determined $1.575 \mathrm{~mm}$. The length of a single pixel of the antenna surface was determined as $\lambda / 10$. The proposed antenna was fed from the center with a $50 \mathrm{ohm} \mathrm{SMA}$ connector.

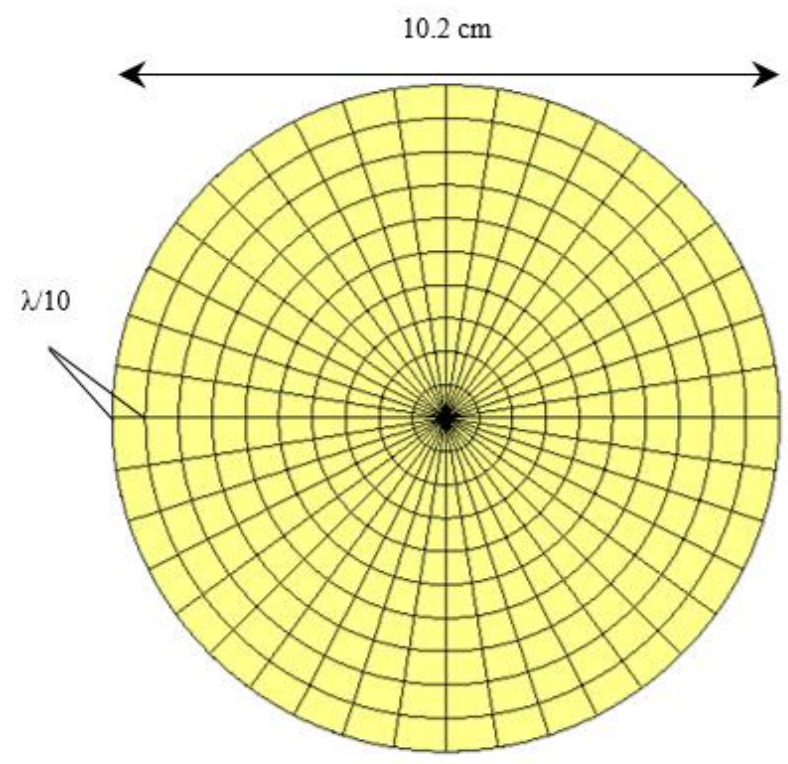

(a)

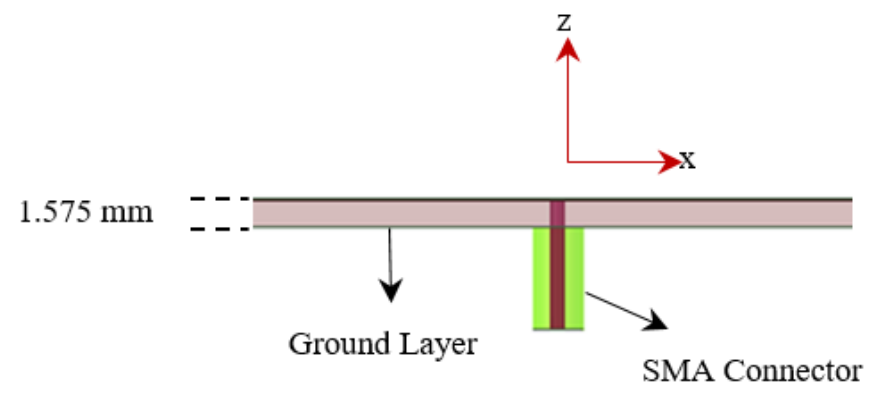

(b)

Fig.1. Antenna geometry: (a) top view of antenna that tailored asymmetric pixels (b) side view of antenna.

\section{B. Genetic Algorithm}

In the first step of the GA process, the initial population is created and the fitness value of each individual in the population is calculated. Then, individuals selected from the population according to the fitness value are crossed and the two parents create a new individual from the individual's genetic codes. The search space is expanded by making random changes in the individual's genes with the mutation in the next step. The rate at which genes are selected for the mutation process is determined by the mutation rate. In the last stage of evolution, the fitness value of the newly created individual is calculated. The fitness of the individual is calculated with the fitness function created according to the determined goal. If the algorithm's maximum iteration count or certain fitness values deemed sufficient for the algorithm are reached, the process ends. Otherwise, the algorithm is restarted depending on the properties of the problem [9].

There are a total of 406 pixels in the structure on the main radiating surface of the antenna (Figure 1a). In the GA we applied, 100 iterations were run for 320 individuals in each generation. The mutation rate in the algorithm was determined as $0.5 \%$. The elitism operator, which ensures that the best chromosomes were not lost during the crossing and mutation stages, was also included in the application. The elitism rate was determined as $1 \%$. The GA has searched automatically for the most suitable structure as it progressed. In this study, we aimed to design both have low input reflection coefficient and high directive antenna.

For this purpose;

$$
\begin{gathered}
\text { fitness }=\max \left(\left[\frac{1}{(V S W R-1)^{m_{1}}}\right]\left(D_{\max }\right)^{m_{2}}\right) \\
m_{1}=\left\{\begin{array}{cc}
0.02, & V S W R \leq 2 \\
0.4, & V S W R>2
\end{array}\right. \\
m_{2}=0.4
\end{gathered}
$$

the above fitness function was created where $D_{\max }$ represents the maximum directivity and VSWR represents voltage standing wave ratio. The weight values $m_{1}$ and $m_{2}$ are very important for the fitness function. These weight values ensured that the VSWR and maximum directivity variables used in the 
fitness function were close to each other. As a result, the Genetic Algorithm searched for an antenna with both directivity and low input reflection coefficient properties at the same time.

Maximum directivity:

$$
D_{\max }=\frac{4 \pi U_{\max }}{P_{\text {rad }}}
$$

expressed in the form. Here, $U_{\max }$ represents the max. radiant intensity and $P_{\text {rad }}$ represents the total radiated power [5]. VSWR's formula [5] is as follows:

$$
V S W R=\frac{1+|\Gamma|}{1-|\Gamma|}
$$

\section{Method of Moments}

So as to calculate the scattered fields from the antenna surface, the surface current densities induced at the antenna surface must be known. The surface current density $\vec{f}(\vec{r})$ is obtained by solving the electric field integral equation (EFIE) created by applying Perfect Electric Conductor (PEC) boundary conditions to conductive surface. The most frequently used basis functions in EFIE's solution with the method of moments are Rao-Wilton-Glisson (RWG) basis functions [22]. Because RWG functions can easily model randomly generated geometric structures with triangles. Due to this feature, full surface analysis of structures with different geometric shapes can be performed by using RWG basis functions. Expressing the surface current density with RWG basis functions using the Galerkin Method [23] is defined as follows:

$$
\vec{J}(\vec{r}) \cong \sum_{n=1}^{N} I_{n} \vec{f}_{n}(\vec{r})
$$

Since the current distributing on the antenna surface is vectorial, the RWG function $\vec{f}_{n}$ is also a vector function. In Galerkin Method, test and basis functions are chosen equally. In this way, since the reciprocity theorem will be provided, high accuracy results are be obtained. One of the most suitable methods that can be used to solve EFIE is Moments Method (MoM) [24]. The method of moments is based on transforming the problem into a matrix equation and solving the resulting matrix equation. The generalized problem;

$$
L\{f(x)\}=g(x)
$$

where $L$ is a linear operator, $g(x)$ is a known feed function and $f(x)$ is the unknown function representing the current distribution. Using the Method of Moments and Galerkin Test Method, this equation;

$$
\left[V_{m}\right]=\left[Z_{m n}\right]\left[I_{n}\right]
$$

turns into the matrix equation where $\left[Z_{m n}\right]$ is the impedance matrix, $\left[I_{n}\right]$ is the unknown coefficients vector and $\left[V_{m}\right]$ is the voltage vector to become.

The most computational step in the simulation of an antenna is the step that involves filling the moment matrix. On the other hand, even a small change in antenna geometry requires full or partial recalculation of the moment matrix. Refilled to the moment matrix each time greatly limits the optimization algorithms to work efficiently in solving the problem. In order to overcome this problem, Direct Matrix Manipulation (DMM) technique, which enables GA optimization to work more efficiently was applied [1]. The DMM method prevents the Zmatrix from being refilled after each evaluation of the fitness function. Thanks to this method, the optimization time is shortened considerably.

\section{RESUlts AND DiscussiOnS}

In the design, firstly, the antenna geometry was created using the software program. In this step, the main geometry of the antenna was drawn and the parts to be included in the optimization were defined. Then, the Z-matrix is calculated. The Z-matrix was calculated taking into account all the metal parts involved in the optimization process on the radiating surface of the antenna. Each metal part was represented by a bit ( 1 or 0 ) on the chromosome. While " 1 " means the presence of metal, setting a bit to " 0 " means that the electric current on that part was zero. This means that the rows and columns of the $\mathrm{Z}$ matrix containing the extracted part were be filled with zeros [1].

Optimization of the antenna was carried out in the MATLAB [25] program and took approximately 50 hours. As a result of optimization process, the best first antenna (BA-1) (Figure 2a), the second best antenna (BA-2) (Figure 2b), the third best antenna (BA-3) (Figure 2c) and (BA-4) (Figure 2d) MATLAB structures of the fourth best antenna were achieved as in Figure 2. The "yellow" color represents the metal regions and the "green" color represents the empty space. Although their shapes were not very different from each other, there were clear differences in the radiation parameters of these antennas.

In this study, the simulations of only the first two antennas with the best fitness value were done using the ANSYS HFSS software program. In addition to these two antennas, an antenna with the same geometric structure but not optimized was simulated and the terminal results (input reflection coefficient, VSWR, group delay) were compared with each other. 


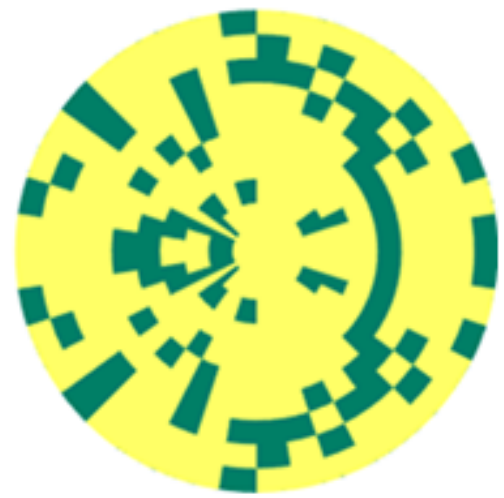

(a)

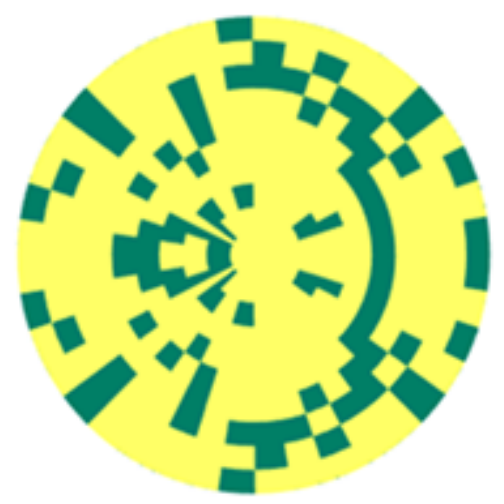

(b)

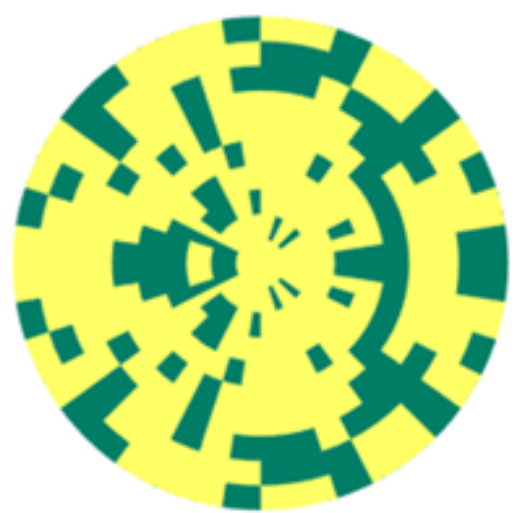

(c)

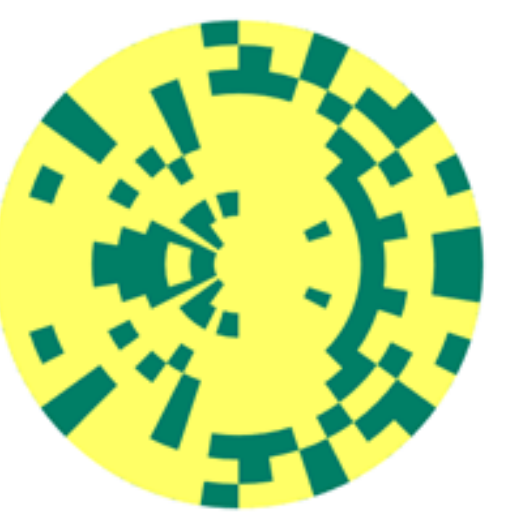

(d)
Fig.2. MATLAB Optimization results (a) the best first antenna (BA-1) (b) the best second antenna (BA-2) (c) the best third antenna (BA-3) (d) the best fourth antenna (BA-4).

\section{A. Surface Current Distribution}

The surface current distributions at $5.8 \mathrm{GHz}$ at the center frequency of the ISM band where the best first antenna (BA-1) and the best second antenna (BA-2) achieved in consequence of the optimization work are shown in Figure 3. In the optimization process, it was aimed to find the best current distribution on the surface according to the specified purpose. As can be seen in Figure 3, while the current on BA-1 was nearly homogeneously distributed on the surface (Figure 3a), on the BA-2 the current was more concentrated in a certain area (Figure 3b).
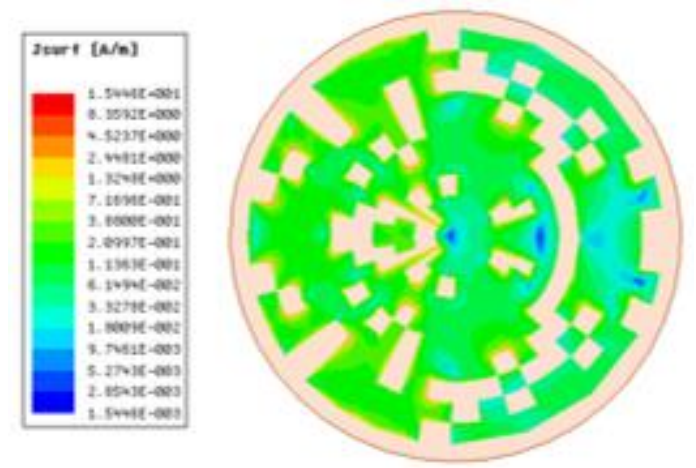

(a)
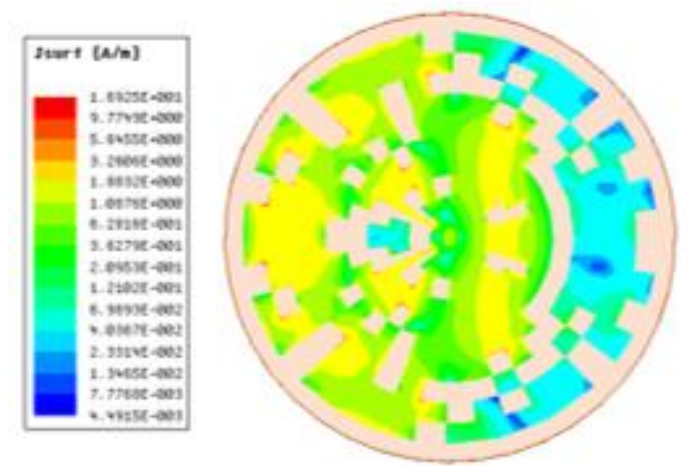

(b)

Fig.3. Surface current distribution of antennas: (a) BA-1 (b) BA-2

\section{B. The Input Reflection Coefficient, Voltage Standing Wave Ratio, and Group Delay}

In Figure 4, the input reflection coefficient of the antenna with the same surface geometry but not optimized (Non-Opt) was compared in addition to the BA-1 and BA-2 antennas. As can be seen, while the Non-Opt antenna did not resonate in the working band, the BA-1's values of $\left|S_{11}\right|$ was $-14.68 \mathrm{~dB}$ at 5.8 $\mathrm{GHz}$ and a $120 \mathrm{MHz}$ bandwidth. The BA-2's values of $\left|S_{11}\right|$ was $-13.29 \mathrm{~dB}$ at $5.8 \mathrm{GHz}$ and a $130 \mathrm{MHz}$. bandwidth. It was determined that the minimum $\left|S_{11}\right|$ value was between 5.75 $\mathrm{GHz}$ and $5.8 \mathrm{GHz}$ for both antennas. To get lower input reflection coefficient values of BA-1 and BA-2 antennas at 5.8 
$\mathrm{GHz}$, the height of the radiating surface, pixel dimensions, and the position of the feeding point can be changed.

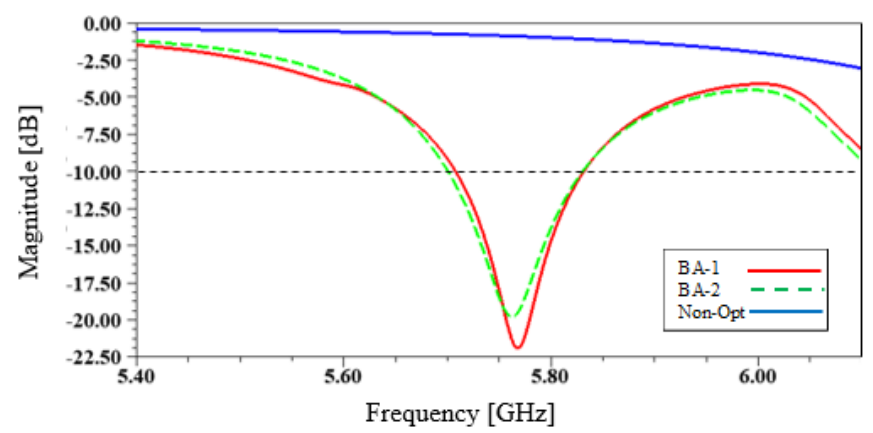

Fig.4. The input reflection coefficient magnitude

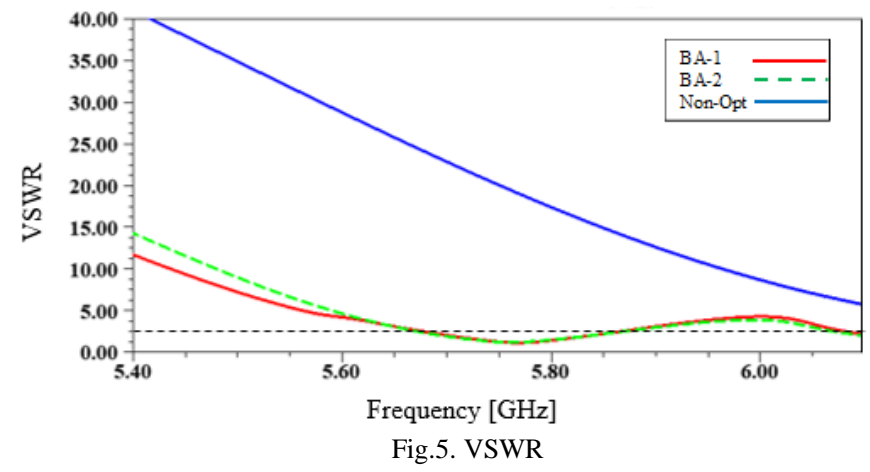

The voltage standing wave ratio (VSWR) simulation results for BA-1, BA-2, and Non-Opt antennas are given in Figure 5. In the operation band, the VSWR values of BA-1 and BA-2 antennas were quite well below the targeted value of 2 . The VSWR value of the Non-Opt antenna was much higher than 2 .

The group delay of proposed antennas designs is given Figure 6. The group delay values of BA-1 and BA-2 antennas in the working band were below $1 \mathrm{~ns}$, which is considered necessary for good communication.

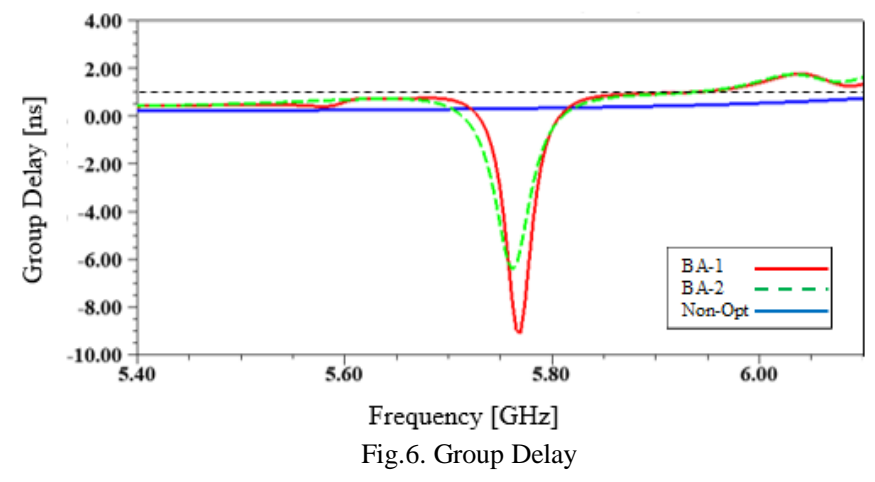

C. Radiation Efficiency, Directivity, Gain, Aperture Efficiency

Resistive losses caused by electric materials and non-perfect metal occur in each antenna. Because of these losses, there is a difference between the input power of the antenna and the power radiated from this antenna. The radiation efficiency of an antenna is defined as the ratio of the antenna's output power to its input power [27].

$$
\eta_{r e}=\frac{P_{o u t}}{P_{\text {in }}}
$$

The radiation efficiency values of the proposed antennas are given in Table I. Accordingly, the radiation efficiency was over $97 \%$ for BA-1 antenna and was over $95 \%$ for BA-2 antenna.

The main fundamental of a directive antenna is the ability to focus power in one given direction and not radiate it in other directions [27]. The normalized directivity patterns for four different frequencies $(5.725,5.75,5.8,5.825 \mathrm{GHz})$ in the 5.8 $\mathrm{GHz}$ ISM band in the E-plane and H-plane of the antennas designed for this purpose are given in Figure 7. As shown in Figure 7, BA-1 and BA-2 antennas had directive characteristics in the E-plane and $\mathrm{H}$-plane for these frequencies in the working band. Although there were some differences in the directivity patterns of these two antennas, it was generally seen that the radiation characteristics of the two antennas were close to each other.
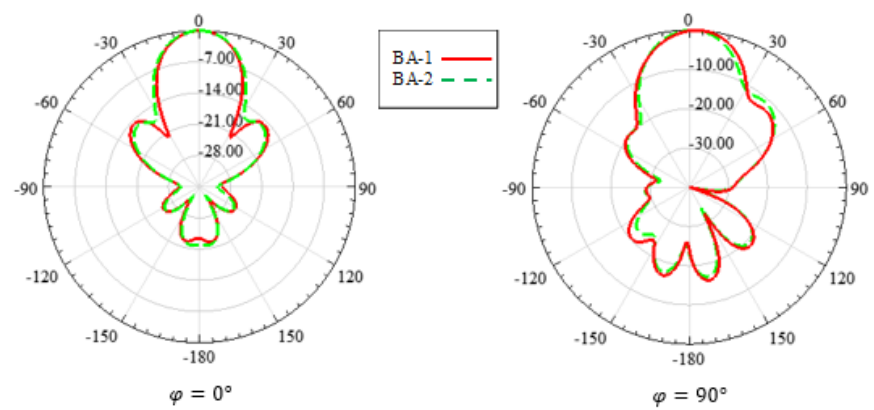

(a)
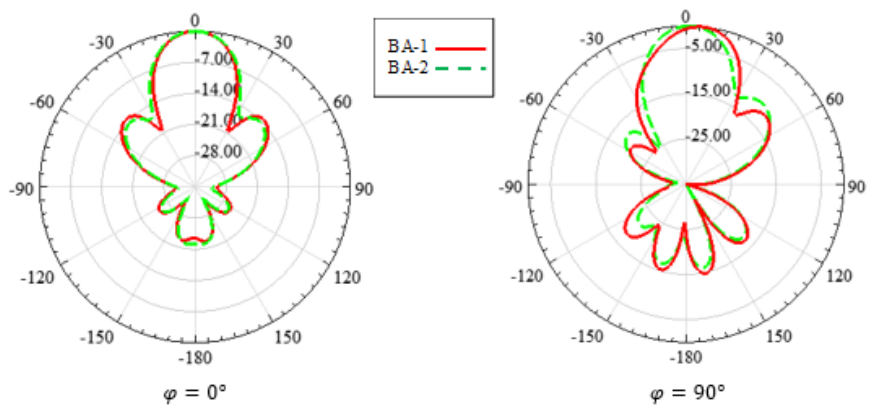

(b)

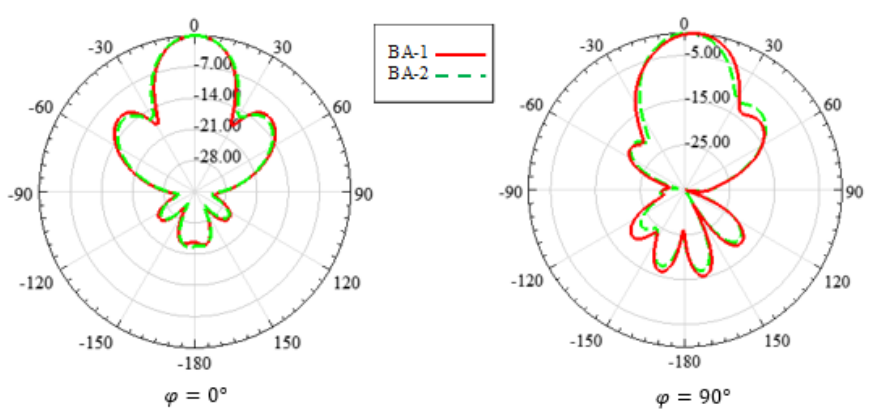

(c) 

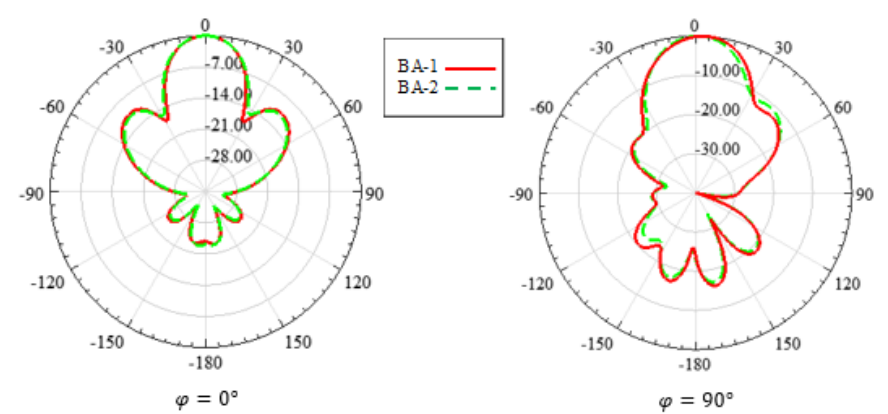

(d)

Fig.7. Normalized directivity patterns in E-plane and $\mathrm{H}$-plane of optimized antennas BA-1 and BA-2 (a) $5.725 \mathrm{GHz}$ (b) $5.75 \mathrm{GHz}$ (c) $5.8 \mathrm{GHz}$ (d) 5.825 $\mathrm{GHz}$

The maximum directivity of the proposed BA-1 antenna in the working band was $15.20 \mathrm{~dB}$ at $5.725 \mathrm{GHz}$ and the maximum directivity for the BA-2 antenna was $15.17 \mathrm{~dB}$ at $5.75 \mathrm{GHz}$. The maximum directivity values for all frequencies are given in Table I.

The directivity of the antenna is only a function of the shape of the antenna's propagation pattern and is not affected by resistive losses in the antenna. Due to a radiation efficiency less than unity an antenna cannot radiate all of its input power, the antenna gain is defined as the product of maximum directivity and radiation efficiency [27]:

$$
G_{\max }=\eta_{\text {re }} D_{\max }
$$

The maximum gain of the proposed antennas BA-1 and BA2 was obtained above $14.5 \mathrm{~dB}$ and close to $15 \mathrm{~dB}$ in the ISM band. The maximum gain values for all frequencies are given in Table I.

In practice, there are many reasons why the directivity of an antenna may not be at its maximum possible value. Non-ideal amplitude and phase characteristics of the aperture field can be given as examples of reasons that reduce the directivity of aperture blockage [27]. The aperture efficiency indicates how much a surface of an antenna effectively radiates, and an aperture efficiency is defined as the ratio of the actual directionality of an antenna to its maximum directivity that cannot be greater than 1 .

$$
\eta_{a p}=\frac{D}{D_{\max }}
$$

TABLE I

\begin{tabular}{|c|c|c|c|c|c|c|c|c|c|c|c|}
\hline No & $\begin{array}{c}f \\
{[G H z]}\end{array}$ & $\begin{array}{c}S_{11} \\
{[d B]}\end{array}$ & VSWR & $\begin{array}{l}S L L \\
{[d B]}\end{array}$ & $\begin{array}{l}\boldsymbol{G}_{\max } \\
{[\boldsymbol{d B i}]}\end{array}$ & $\begin{array}{l}D_{\max } \\
{[d B i]}\end{array}$ & $\begin{array}{c}\text { Rad Eff. } \\
{[\%]}\end{array}$ & $\begin{array}{l}\boldsymbol{\varepsilon}_{a p} \\
{[\%]}\end{array}$ & $\begin{array}{c}\boldsymbol{H P B W} \\
{\left[^{\circ}\right]}\end{array}$ & $\begin{array}{l}\text { Gd } \\
{[\mathrm{ns}]}\end{array}$ & $\begin{array}{c}B W \\
{[M H z]}\end{array}$ \\
\hline \multirow[t]{4}{*}{$B A-1$} & 5.725 & -12.38 & 1.63 & -14.68 & 15.10 & 15.20 & 97.62 & 81.96 & 26.54 & 0.16 & \multirow{4}{*}{120} \\
\hline & 5.75 & -18.01 & 1.29 & -13.36 & 14.95 & 15.05 & 97.64 & 79.18 & 26.03 & -3.08 & \\
\hline & 5.8 & -14.68 & 1.45 & -11.30 & 14.73 & 14.83 & 97.74 & 75.40 & 25.37 & -0.57 & \\
\hline & 5.825 & -10.73 & 1.82 & -10.55 & 14.67 & 14.77 & 97.75 & 74.34 & 25.11 & 0.57 & \\
\hline \multirow[t]{4}{*}{$B A-2$} & 5.725 & -13.50 & 1.54 & -15.57 & 14.95 & 15.15 & 95.43 & 79.28 & 27.95 & -0.65 & \multirow{4}{*}{130} \\
\hline & 5.75 & -18.48 & 1.27 & -14.46 & 14.97 & 15.17 & 95.52 & 79.63 & 27.43 & -4.55 & \\
\hline & 5.8 & -13.79 & 1.51 & -12.17 & 14.89 & 15.09 & 95.52 & 78.16 & 26.40 & -0.57 & \\
\hline & 5.825 & -10.53 & 1.85 & -11.07 & 14.78 & 14.98 & 95.41 & 76.14 & 25.89 & 0.41 & \\
\hline
\end{tabular}

SIMULATION RESULTS

High aperture efficiency was achieved for both proposed antennas. The maximum aperture efficiencies of the proposed BA-1 and BA-2 antennas were obtained at $5.725 \mathrm{GHz}$ with $81.96 \%$ and $79.63 \%$ at $5.75 \mathrm{GHz}$, respectively. The aperture efficiency values for all frequencies are given in Table I.

\section{CONCLUSION}

In this article, two optimal antennas were designed using GA / MoM approach in the 5.8 GHz ISM band. The simulation results of the best first and best second antennas were compared with each other. The gains of both antennas were higher than $14.7 \mathrm{~dB}$ at $5.8 \mathrm{GHz}$ center frequency. The input reflection coefficient and side lobe level (SLL) values were below -10 dB for both antennas.

In this study, it was shown that antenna designs with different geometries and aimed properties can be made with the asymmetric pixelation approach that was introduced for the first time. It was emphasized that by choosing a pixel length very short, the antenna surface can be examined in more detail. However, in this case, it should be noted that the dimensions of the $\mathrm{Z}$ matrix will increase and this will result in an increase in optimization time.

\section{REFERENCES}

[1] J. M. Johnson and Y. Rahmat-Samii, "Genetic algorithms and method of moments (GA/MOM) for the design of integrated antennas," in IEEE Transactions on Antennas and Propagation, vol. 47, no. 10, pp. 16061614, Oct. 1999, doi: 10.1109/8.805906.

[2] N. Jin and Y. Rahmat-Samii, "Parallel particle swarm optimization and finite- difference time-domain (PSO/FDTD) algorithm for multiband and wide-band patch antenna designs," in IEEE Transactions on Antennas and Propagation, vol. 53, no. 11, pp. 3459-3468, Nov. 2005, doi: 10.1109/TAP.2005.858842. 
[3] K. Kunz and R. Luebbers, The Finite Difference Time Domain Method for Electromagnetics. CRC Press, 1993.J.G. Van Bladel, Electromagnetic Fields, John Wiley \& Sons, 2007, p.1176.

[4] J. L. Volakis, A. Chatterjee, and L. C. Kempel, Finite Element Method for Electromagnetics. IEEE Press, 1998.

[5] C. A. Balanis, Advanced Engineering Electromagnetics. John Wiley and Sons, 1989.

[6] N. Jin and Y. Rahmat-Samii, "Advances in Particle Swarm Optimization for Antenna Designs: Real-Number, Binary, Single-Objective and Multiobjective Implementations," in IEEE Transactions on Antennas and Propagation, vol. 55, no. 3, pp. 556-567, March 2007, doi: 10.1109/TAP.2007.891552.

[7] O. Quevedo-Teruel and E. Rajo-Iglesias, "Ant Colony Optimization in Thinned Array Synthesis With Minimum Sidelobe Level," in IEEE Antennas and Wireless Propagation Letters, vol. 5, pp. 349-352, 2006, doi: 10.1109/LAWP.2006.880693.

[8] W. Wang, S. Gong, X. Wang, Y. Guan and W. Jiang, "Differential Evolution Algorithm and Method of Moments for the Design of LowRCS Antenna," in IEEE Antennas and Wireless Propagation Letters, vol. 9, pp. 295-298, 2010, doi: 10.1109/LAWP.2010.2047837.

[9] Haupt, R. L. \& Werner, D. H. Genetic Algorithms in Electromagnetics (Wiley, Boca Raton, 2007).

[10] Bilotti, F., Castellana, F. and Vegni, L. (2002), Multi-frequency patch antenna design via the method of moment and genetic algorithm. Microw. Opt. Technol. Lett., 35: 184-186. https://doi.org/10.1002/mop.10551

[11] J. Leonardo Araque Quijano and G. Vecchi, "Optimization of an Innovative Type of Compact Frequency-Reconfigurable Antenna," in IEEE Transactions on Antennas and Propagation, vol. 57, no. 1, pp. 918, Jan. 2009, doi: 10.1109/TAP.2008.2009649.

[12] Rengarajan, S.R. (2013), Genetic algorithm optimization of a planar slot array using full wave method-of-moments analysis. Int $\mathrm{J} R F$ and Microwave Comp Aid Eng, 23: 430-436. https://doi.org/10.1002/mmce.20730

[13] S. Song and R. D. Murch, "An Efficient Approach for Optimizing Frequency Reconfigurable Pixel Antennas Using Genetic Algorithms," in IEEE Transactions on Antennas and Propagation, vol. 62, no. 2, pp. 609-620, Feb. 2014, doi: 10.1109/TAP.2013.2293509.

[14] Khuntia, B., Pattnaik, S.S., Panda, D.C., Neog, D.K., Devi, S. and Dutta, M. (2005), Genetic algorithm with artificial neural networks as its fitness function to design rectangular microstrip antenna on thick substrate. Microw. Opt. Technol. Lett., 44: 144-146. https://doi.org/10.1002/mop.20570

[15] A. H. Hussein, H. H. Abdullah, A. M. Salem, S. Khamis and M. Nasr, "Optimum Design of Linear Antenna Arrays Using a Hybrid MoM/GA Algorithm," in IEEE Antennas and Wireless Propagation Letters, vol. 10, pp. 1232-1235, 2011, doi: 10.1109/LAWP.2011.2174189.

[16] S. Chakravarty, R. Mittra and N. R. Williams, "Application of a microgenetic algorithm (MGA) to the design of broadband microwave absorbers using multiple frequency selective surface screens buried in dielectrics," in IEEE Transactions on Antennas and Propagation, vol. 50, no. 3, pp. 284-296, March 2002, doi: 10.1109/8.999618.

[17] L. Alatan, M. I. Aksun, K. Leblebicioglu and M. T. Birand, "Use of computationally efficient method of moments in the optimization of printed antennas," in IEEE Transactions on Antennas and Propagation, vol. 47, no. 4, pp. 725-732, April 1999, doi: 10.1109/8.768813.

[18] Zucchi, M., Giordanengo, G., Righero, M. et al. First demonstration of machine-designed ultra-flat, low-cost directive antenna. Sci Rep 10, 10506 (2020). https://doi.org/10.1038/s41598-020-67354-2

[19] B. Karaosmanoğlu, S. Güler, H. İbili and Ö. Ergül, "Inkjet-printed pixel antennas with hexagonal cells," 2017 11th European Conference on Antennas and Propagation (EUCAP), Paris, 2017, pp. 2013-2016, doi: 10.23919/EuCAP.2017.7928488.

[20] "IEEE Standard for Definitions of Terms for Antennas," in IEEE Std 145-2013 (Revision of IEEE Std 145-1993), vol., no., pp.1-50, 6 March 2014, doi: 10.1109/IEEESTD.2014.6758443.

[21] S. Rao, D. Wilton and A. Glisson, "Electromagnetic scattering by surfaces of arbitrary shape," in IEEE Transactions on Antennas and Propagation, vol. 30, no. 3, pp. 409-418, May 1982, doi: 10.1109/TAP.1982.1142818.

[22] D. Schaubert, D. Wilton and A. Glisson, "A tetrahedral modeling method for electromagnetic scattering by arbitrarily shaped inhomogeneous dielectric bodies," in IEEE Transactions on Antennas and Propagation, vol. 32, no. 1, pp. 77-85, January 1984, doi: 10.1109/TAP.1984.1143193.

[23] A. F. Peterson, D. R. Wilton and R. E. Jorgenson, "Variational nature of Galerkin and non-Galerkin moment method solutions," in IEEE Transactions on Antennas and Propagation, vol. 44, no. 4, pp. 500-503, April 1996, doi: 10.1109/8.489301.

[24] Gibson, W. C. The Method of Moments in Electromagnetics (Chapman \& Hall/CRC, Boca Raton, 2008).

[25] https://www.mathworks.com/products/matlab.html

[26] https://www.ansys.com/products/electronics/ansys-hfss

[27] Pozar, D.M. (2011) Microwave Engineering. 4th Edition. Wiley, New York.

\section{BIOGRAPHY}

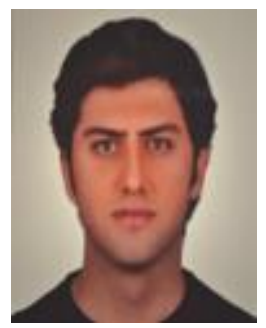

SINAN DOĞUSAN He was born in Malatya, Turkey, 1989. He received the BSc. Degree in Department of Electrical and Electronics Engineering from Yeditepe University in 2016. Currently, he is continuing his MSc. study at Inonu University. While studying at Inonu University, he worked a term as a Visiting Researcher at Antenna and Electromagnetic Compatibility Laboratory (LACE) in Politecnico di Torino. His research interests are Electromagnetic Fields and Applications, Antennas, Microwave and RF Engineering. 